\title{
Health Literacy in Veterans with Spinal Cord Injury Referred to Khatam Alanbia Hospital, Tehran, Iran
}

\section{Naser Sedghi Goyaghaj ${ }^{1}$, Zahra seidi ${ }^{2}$, Zahra Darvishi ${ }^{3 *}$, Fatemeh Mokhtari ${ }^{3}$}

${ }^{1}$ Shefa Neuroscience Research Center, Khatam Alanbia Hospital, Tehran, Iran

${ }^{2}$ Department of Nursing, School of Nursing and Midwifery, Islamic Azad University of Medical Sciences, Tehran, Iran ${ }^{3}$ Department of Pediatrics, School of Nursing and Midwifery, Shahid Beheshti University of Medical Sciences, Tehran, Iran

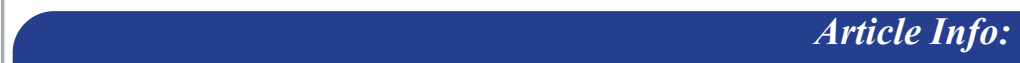

\section{A BSTRACT}

Introduction: Despite the importance of health literacy in chronic diseases and its impact on health behaviors, its importance in patients with spinal cord injuries has been neglected. This study aimed to determine health literacy in veterans with spinal cord injury referred to Khatam Alanbia Hospital, Tehran, Iran. Materials and Methods: This study was a descriptive-analytical cross-sectional study. All veterans with paraplegia following spinal cord injury, who were referred to Khatam Alanbia Hospital in 2018 were our statistical research community. 200 veterans were selected and examined through the purposive sampling method based on inclusion criteria. Demographic characteristics of veterans and Iranian adult health literacy questionnaires were used to collect data. Results: The overall health literacy score was inadequate in $38 \%$ of the veterans. The mean score of health literacy was $51.4 \pm$ 8.8. The aspect of access to health literacy promotion resources received the highest score and there was a significant relationship between health literacy, education, and occupation. Conclusion: The data revealed that the level of health literacy of veterans with spinal cord injury is not desirable. Improving health literacy in these patients reduces the complications and promotes self-care behaviors.

*Corresponding Author: Zahra Darvishi

E-mail: zahradarvishi6973@gmail.com 


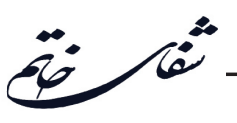

\title{
بررسى سطح سواد سلامت جانبازان ضايعات نخاعى مر اجعهكننده به بيمارستان خاتم الانبياء تهران، ايران
}

\author{
ناصر صدقى توى آقاج'، زهرا صيدى ‘، زهرا درويشى "ّ:، فاطمه مختارى"
}

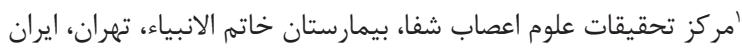

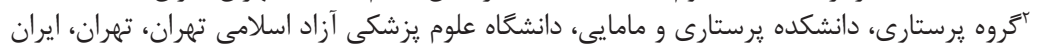

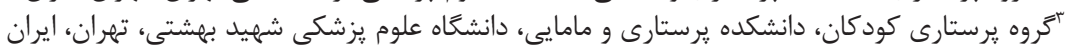

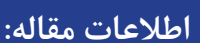

يذيرش: م آبان

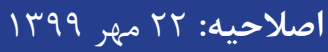

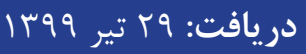

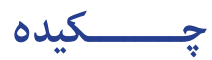

مقدمه: باوجود اهميت سطح سواد سلامت در بيمارى هاى مزمن و و تاثير آن بر انجام رفتار آنار هاى سلامت، توجه

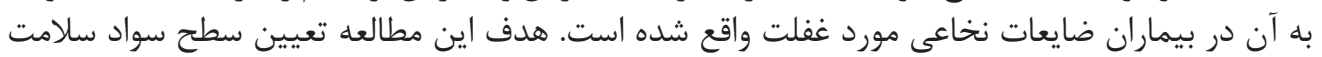

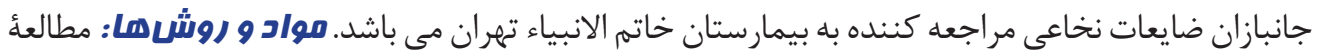

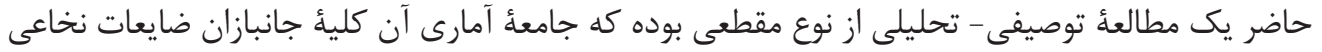

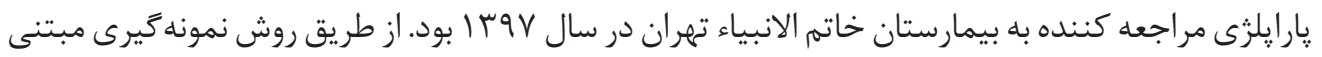

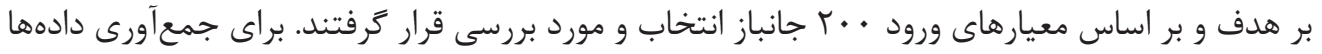

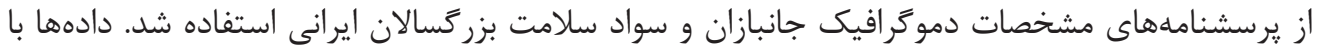

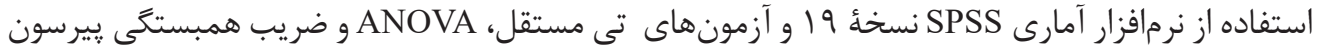

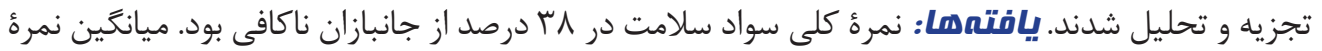

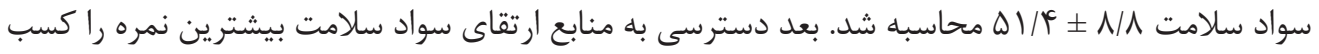

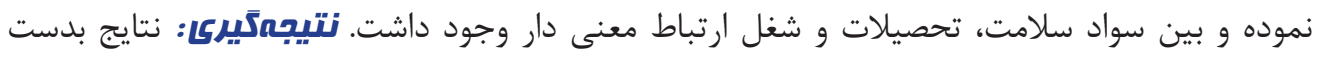

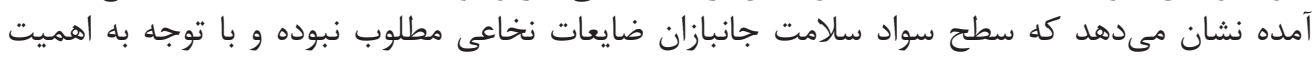

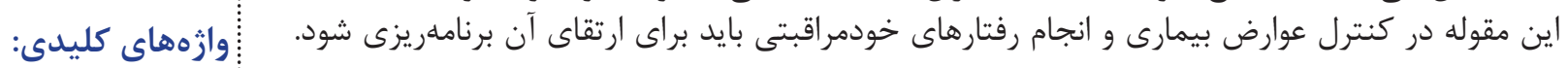

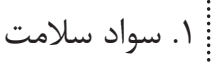

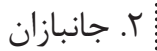

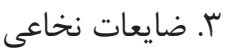

$$
\text { *ويسنده مسئول: زهرا درويشى }
$$

zahradarvishi6973@gmail.com : يست الكترونيك 


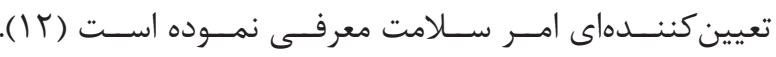

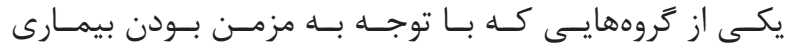

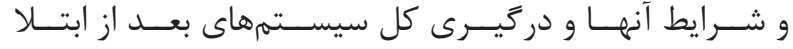

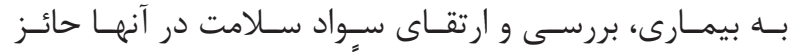

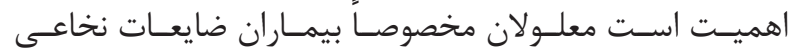

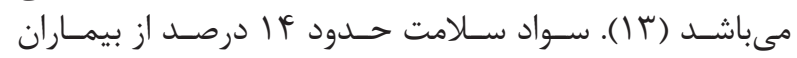

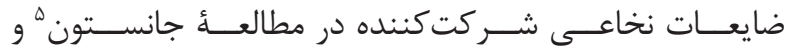

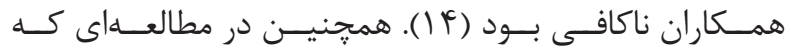

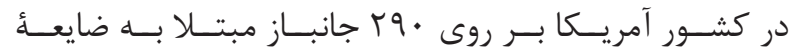

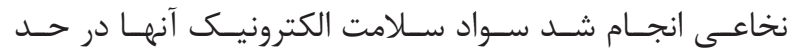

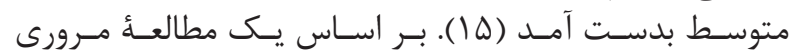

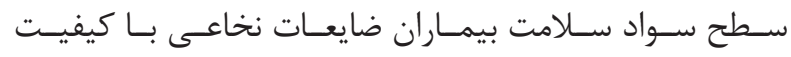

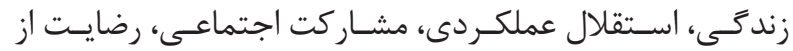

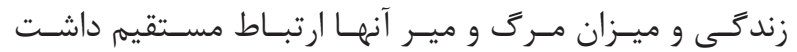

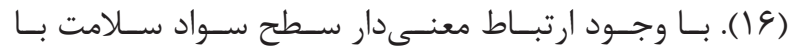

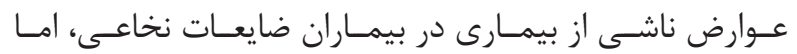

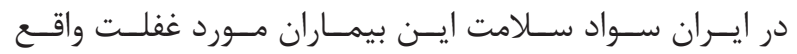

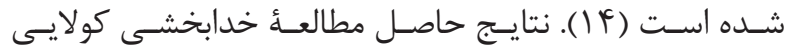

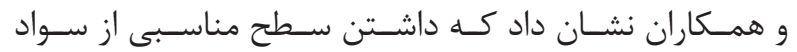

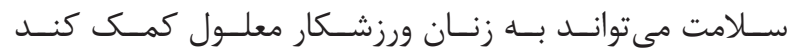

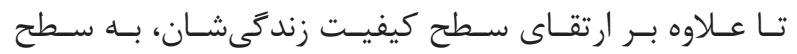

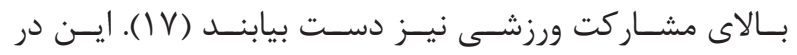

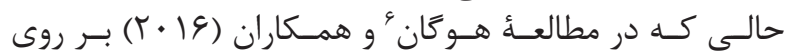

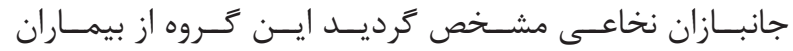

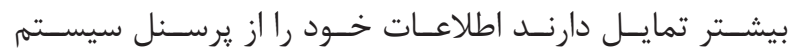

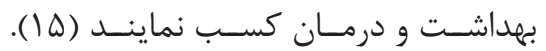

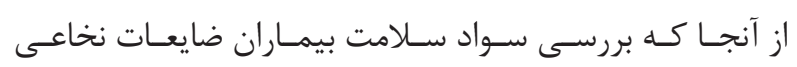

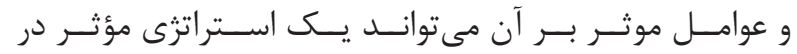

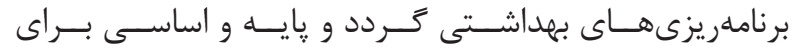

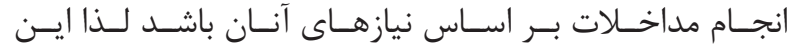

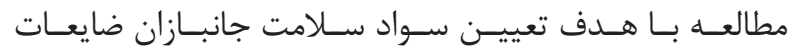

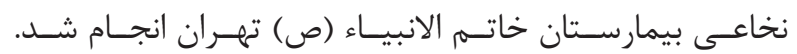

\section{مواد و روشها - - ماد}

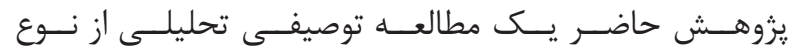

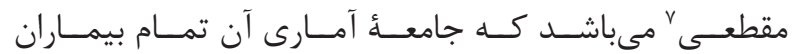

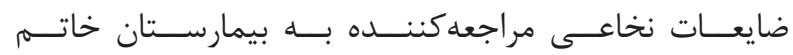

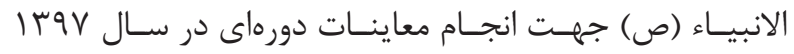

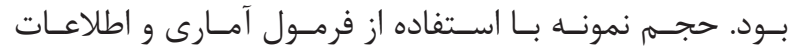

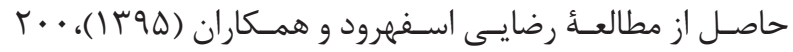

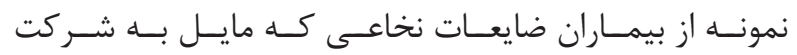

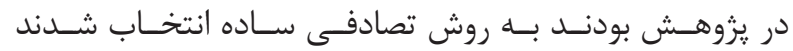

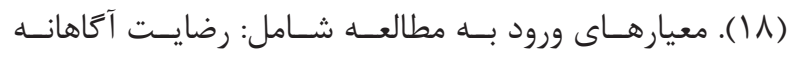

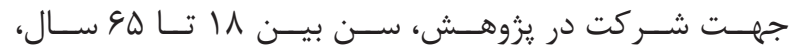

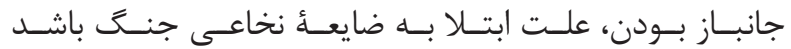

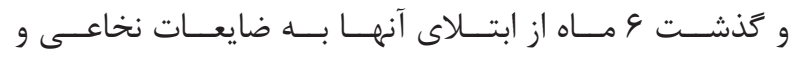

${ }^{1}$ Spinal Cord Injury

${ }^{2}$ Quality of Life

${ }^{3}$ Health Literacy

${ }^{4}$ World Health Organization; WHO

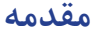

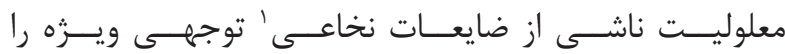

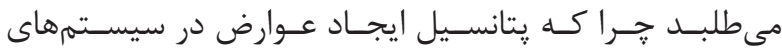

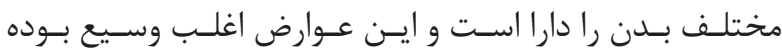

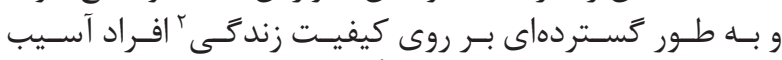

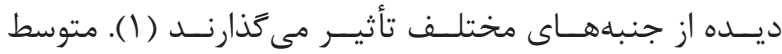

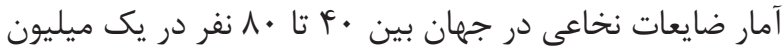

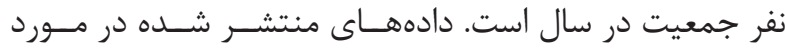

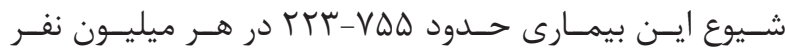

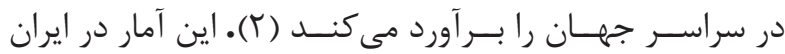

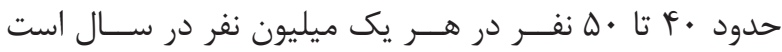

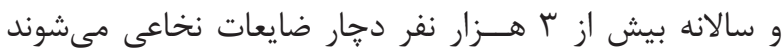

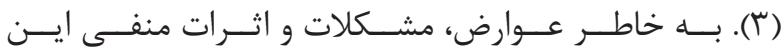

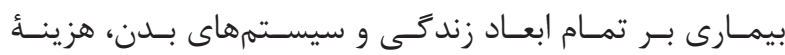

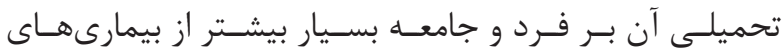

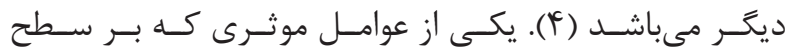

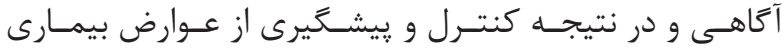

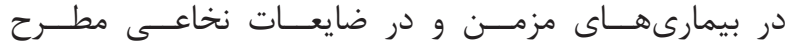

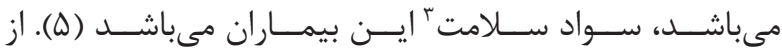

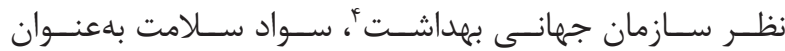

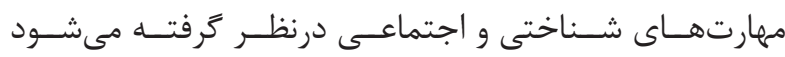

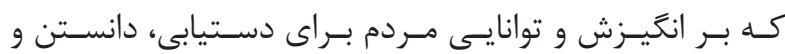

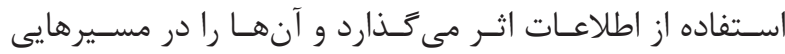

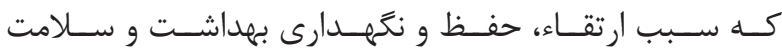

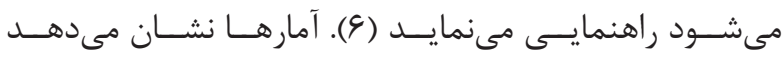

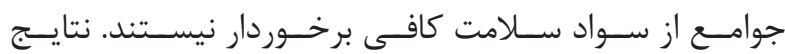

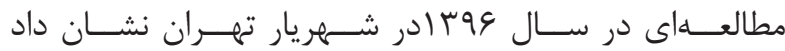

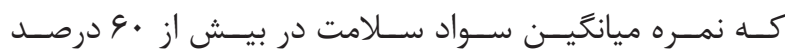

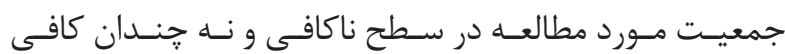

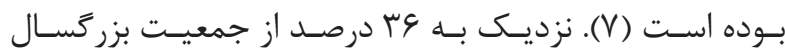

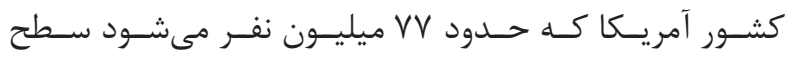

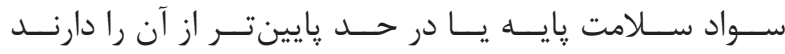

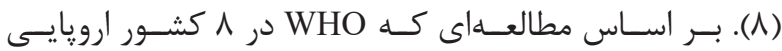

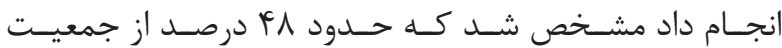

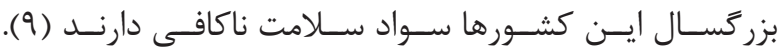

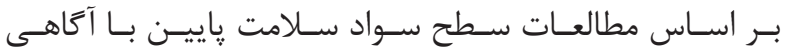

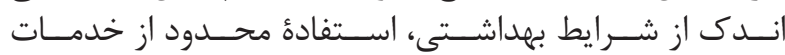

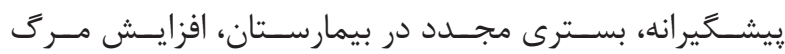

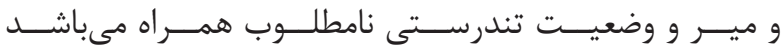

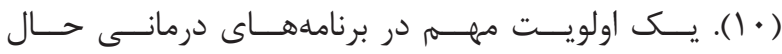

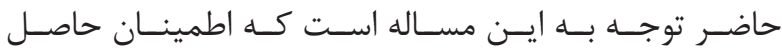

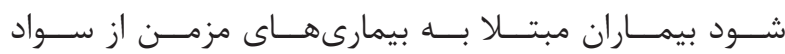

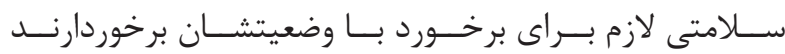

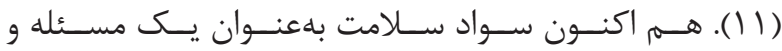

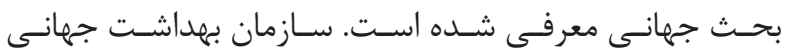

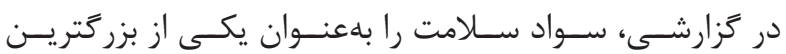

${ }^{5}$ Johnston

${ }^{6}$ Hogan

${ }^{7}$ Descriptive analytical cross-sectional study 


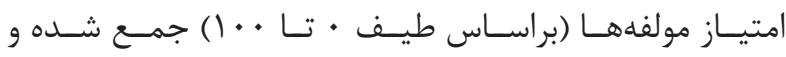

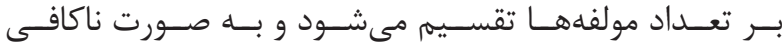

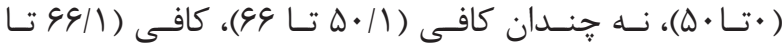

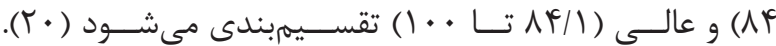

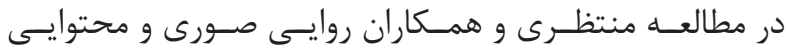

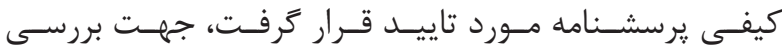

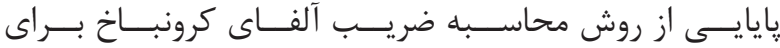

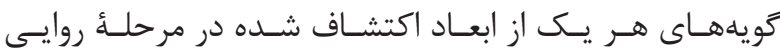

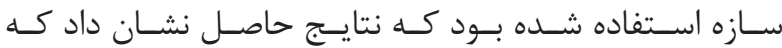

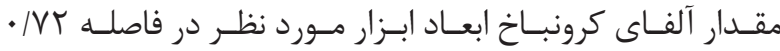

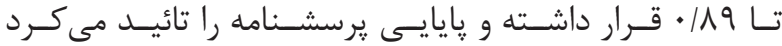

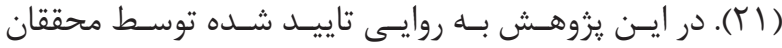

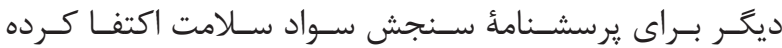

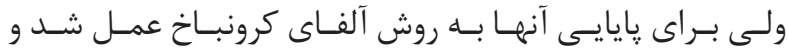

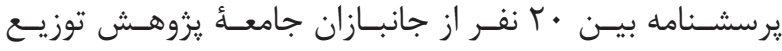

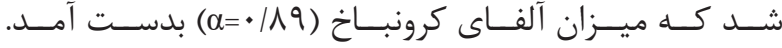

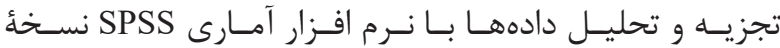

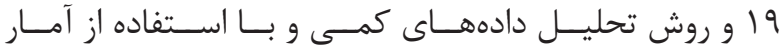

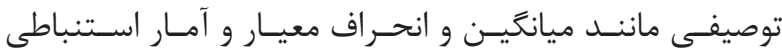

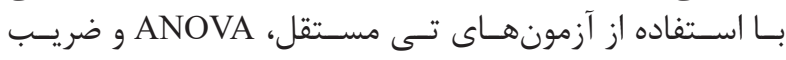

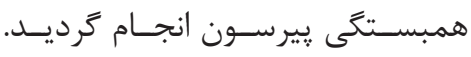

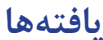

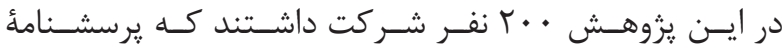

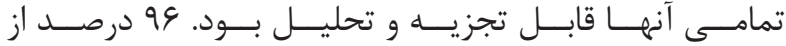

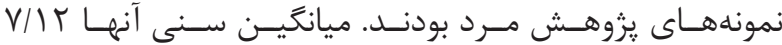

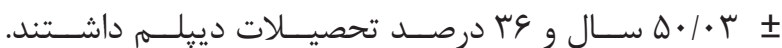

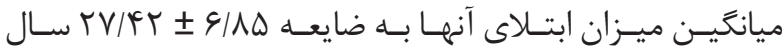

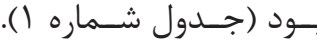

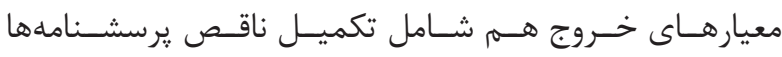

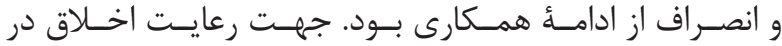

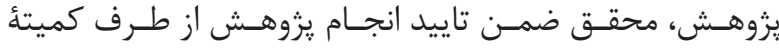

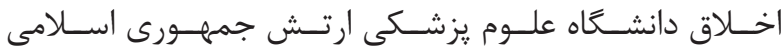

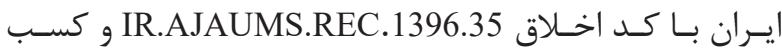

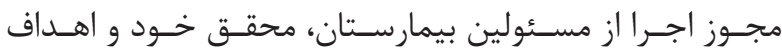

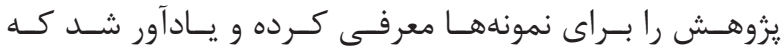

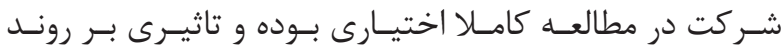

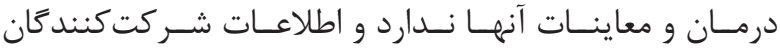

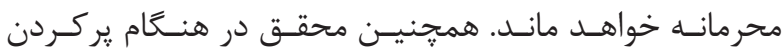

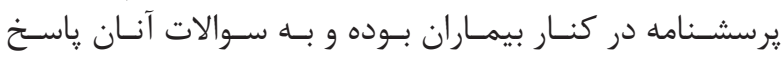

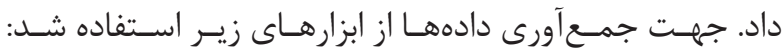

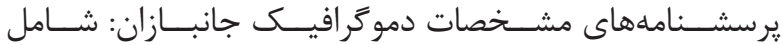

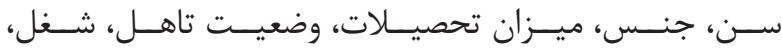

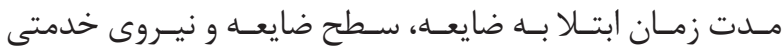

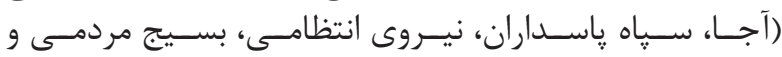

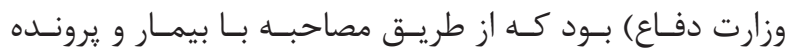

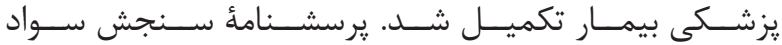

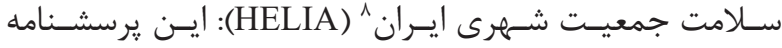

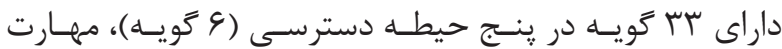

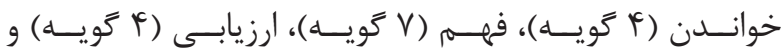

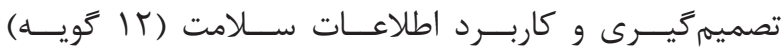

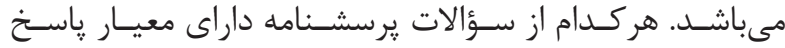

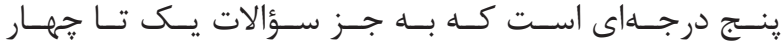

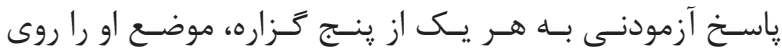

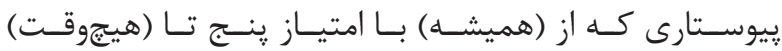

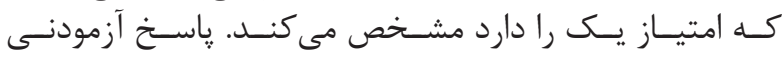

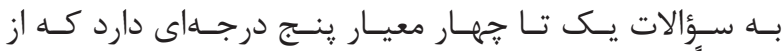

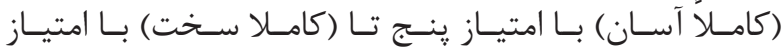

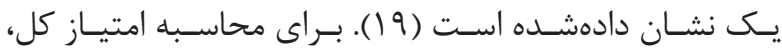

جدول ا- ويثزى هاى فردى و بيمارى جانبازان

\begin{tabular}{|c|c|c|c|}
\hline درصد & تعגداد & \multicolumn{2}{|c|}{ متثبر } \\
\hline 96 & 195 & مود & \multirow{2}{*}{ جنست } \\
\hline$f$ & ᄉ & زن & \\
\hline rt & ef & زيوديهيلم & \multirow{3}{*}{ تحصيلات } \\
\hline F9 & ar & ديبلمت & \\
\hline tr & $f f$ & دان=تعاهيى & \\
\hline IT & th & مجرد & \multirow{2}{*}{ تاعل } \\
\hline 从ᄉ & IVe & متاهل & \\
\hline V/o & 10 & بيكار & \multirow{5}{*}{ شتل } \\
\hline 19 & rr & آزاد & \\
\hline 19 & r人 & كارمند & \\
\hline$\Delta f / \Delta$ & 1.9 & بازنثشته & \\
\hline$r$ & 9 & خانهدار & \\
\hline$\Delta / \Delta$ & 11 & $1 \mathrm{~N}-\mathrm{T \Delta}$ & \multirow{3}{*}{ ست } \\
\hline erio & $1 T 0$ & $r s-\Delta$. & \\
\hline$r T$ & sf & $\Delta 1-9 \Delta$ & \\
\hline$F+10$ & A) & سينهاى & \multirow{2}{*}{ سطح ضايعه } \\
\hline$\Delta Q / \Delta$ & 119 & كمرى & \\
\hline ri & et & آجا & \multirow{5}{*}{ تيروى خدمتى } \\
\hline TI & ft & نس & \\
\hline fis & 9 & تيروى اثتظامي & \\
\hline$\wedge$ & 19 & وزأرت دماع & \\
\hline TANO & 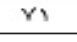 & يسبيج مردميى & \\
\hline
\end{tabular}

${ }^{8}$ Health Literacy for Iranian Adults; HELIA

${ }^{9}$ Hogan 


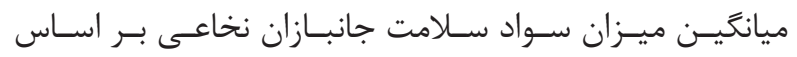

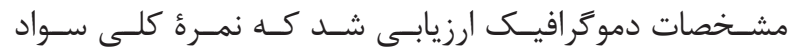

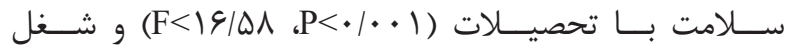

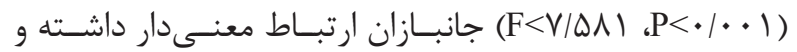

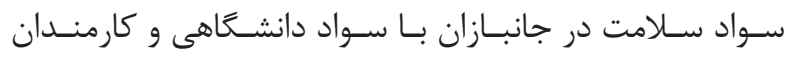
بالاتـر بـود (جــدول شـماره س).

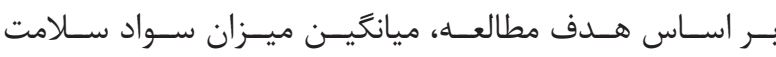

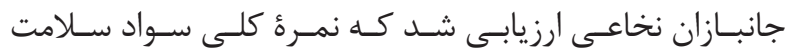

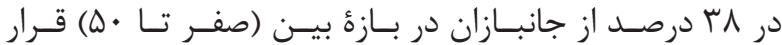

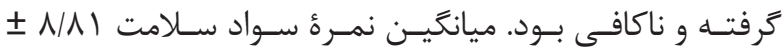

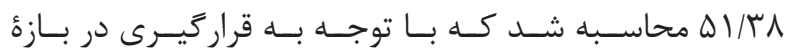

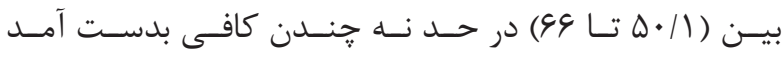

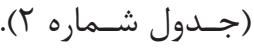

جدول r- ميانكين و انحراف معيار نمرات سواد سلامت جانبازان

\begin{tabular}{|c|c|c|c|c|}
\hline انحراف معيار & ميانگين & بيشـينه & كمينه & متغير \\
\hline$r \cdot|q|$ & $\Delta T / K \wedge$ & $1 \cdots$ & $1 T / \Delta$ & مهارت خواندن \\
\hline 19/r 19 & $9 \cdot 199$ & १人 & $19 / 99$ & دسترسى \\
\hline $1 \mathrm{~V} / 1 \wedge$ & Fヘ/rV & $1 \cdots$ & tr & فهم و درك \\
\hline th/4t & $R \cdot / T_{1}$ & 91 & G/TQ & ارزيابى \\
\hline $19 / 14$ & $\Delta \Delta$ & $1 \cdots$ & re & تصميسمَيرى و رفتار \\
\hline 人/ᄉ I & ه) & $V \varepsilon / \mathcal{R}$ & $r V / e$ & كل سواد سلامت \\
\hline
\end{tabular}

جدول r - ارتباط بين مشخصات دموكر افيك جانبازان و نمرءٔ سواد سلامت

\begin{tabular}{|c|c|c|c|}
\hline آزمون آمارى & اثحراف معيار \ مياثخين & \multicolumn{2}{|c|}{ متثير } \\
\hline \multirow{2}{*}{$\begin{array}{l}\mathrm{P}<+/ f \mid 9 \\
\mathrm{t}<+|\wedge|+\end{array}$} & $\Delta 1 / \uparrow q \pm N(\lambda)$ & مرد & \multirow[t]{2}{*}{ جنسيت } \\
\hline & FNVT \pm NNG & ن & \\
\hline \multirow{3}{*}{$\begin{array}{l}\mathrm{P}<+/ \cdots 1 \\
\mathrm{~F}<|\xi| \Delta \lambda\end{array}$} & fq/19 $\pm \mathrm{V} / \mathrm{rr}$ & زيردييلهم & \multirow[t]{3}{*}{ 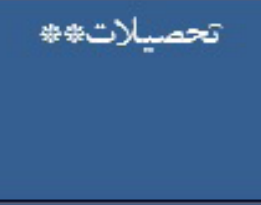 } \\
\hline & $f q / V) \pm N \cdot I$ & ديبلم & \\
\hline & $\Delta N / T \pm 1 / / N$ & دانشثُاهى & \\
\hline \multirow{2}{*}{$\begin{array}{l}\mathrm{P}<. / 190 \\
\mathrm{t}<1 / 499\end{array}$} & $\Delta r / V+N \cdot N$ & مجرد & \multirow[t]{2}{*}{ تاهل } \\
\hline & $\Delta / / \cdot 9 \pm 9 / 01$ & متاهل & \\
\hline \multirow{5}{*}{$\begin{array}{l}\mathrm{P}<+/ \cdots 1 \\
\mathrm{~F}<\mathrm{V} / \Delta \wedge)\end{array}$} & $F g / r) \pm q / r r$ & بيكار & \multirow[t]{5}{*}{ شئ } \\
\hline & $f q / r+ \pm f / \cdot q$ & آزاد & \\
\hline & $\Delta N+\Lambda \pm V / f 1$ & كارمند & \\
\hline & $\Delta \cdot / 9 \cdot \pm 9 / 01$ & بازئشستـ & \\
\hline & $F V / \Delta \pm q / V r$ & خائنددار & \\
\hline \multirow{2}{*}{$\begin{array}{l}\mathrm{P}<+|| \wedge \mid \\
\mathrm{t}<\left|/ \mathrm{T}^{\prime}\right|\end{array}$} & $\Delta \cdot / r q \pm q / \cdot \Delta$ & سينـلهاى & \multirow[t]{2}{*}{ سحلح ضايعها } \\
\hline & $\Delta T / I T \pm N G F$ & كمرى & \\
\hline \multirow{5}{*}{$\begin{array}{l}\mathrm{P}<\cdot / \Delta f F \\
\mathrm{~F}<\cdot / \gamma \cdot r\end{array}$} & $\Delta / / 91 \pm N T \Delta$ & آجا & \multirow[t]{5}{*}{ ثبروى خدمتى } \\
\hline & $\Delta T / \lambda T \pm 9 / 9 F$ & سياه & \\
\hline & F/99 \pm F/VD & نيروى انتظامى & \\
\hline & $\Delta / / 1 \cdot \pm 1 / / T F$ & وزارت دفاع & \\
\hline & $\Delta \cdot / 9 \Delta \pm 9 / 4 F$ & بسيتج مردمى & \\
\hline $\begin{array}{l}\mathrm{P}<+/ \| \wedge \mathrm{V} \\
\mathrm{r}<+1+91\end{array}$ & $\Delta \cdot / \cdot r \pm V / 1 r$ & انحراف معيار ـ مياتُخين & سن \\
\hline
\end{tabular}




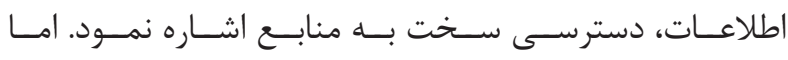

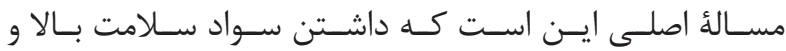

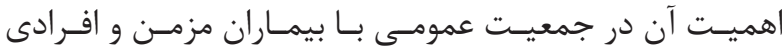

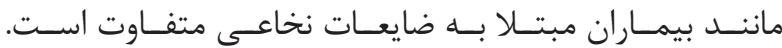

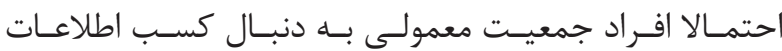

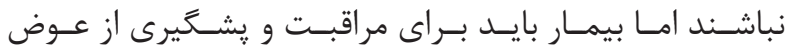

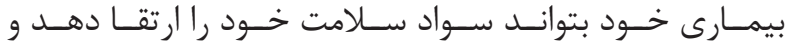

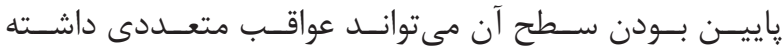

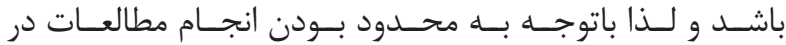

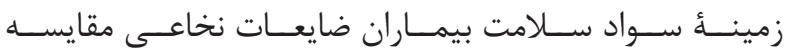

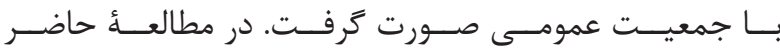

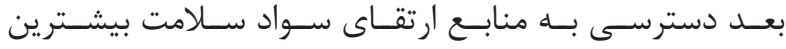

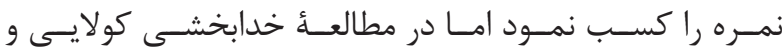

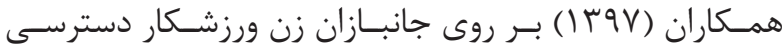

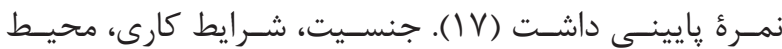

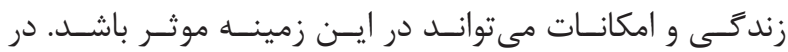

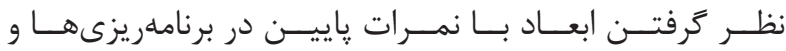

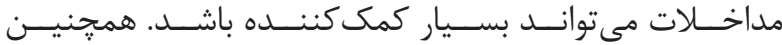

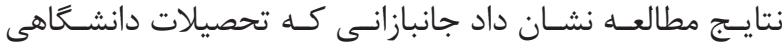

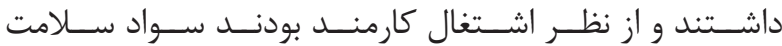

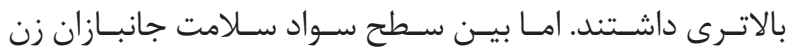

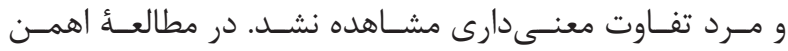

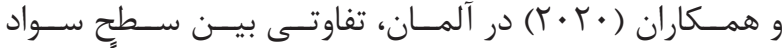

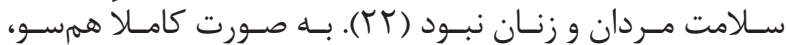

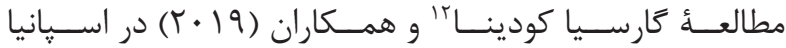

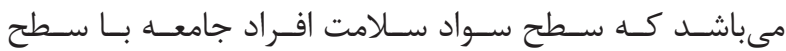

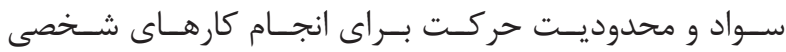

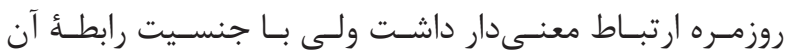

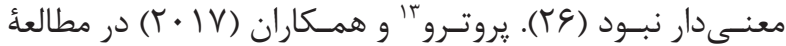

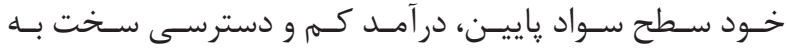

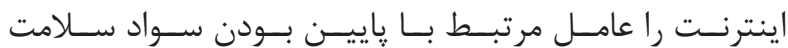

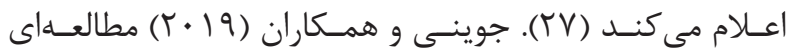

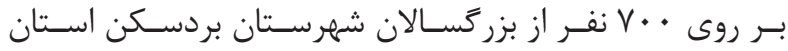

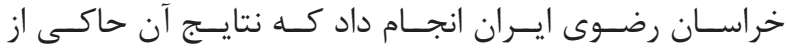

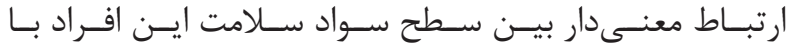

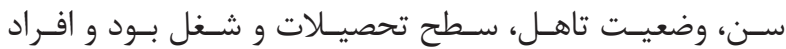

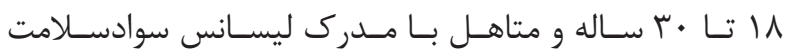

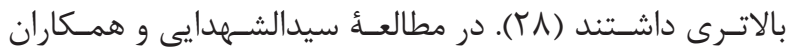

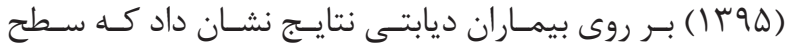

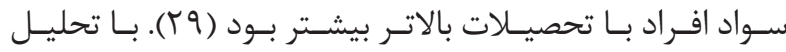

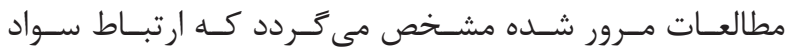

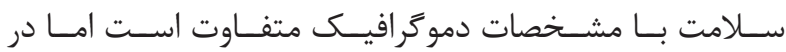

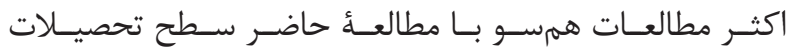

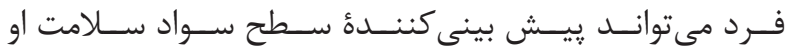

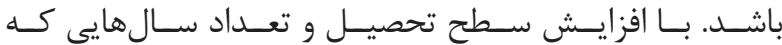

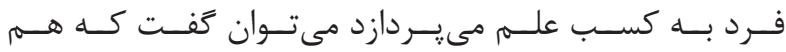

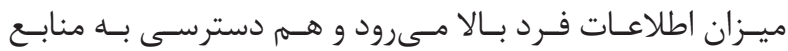

${ }^{10}$ Johnston

${ }^{11}$ Ehmann

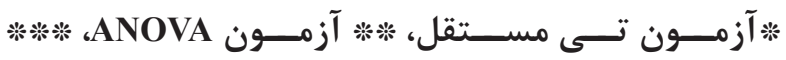

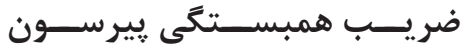

بحث و نتيجه تيرى

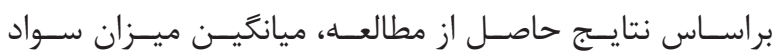

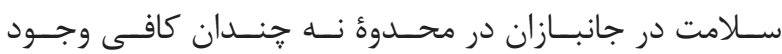

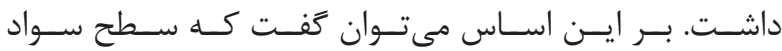

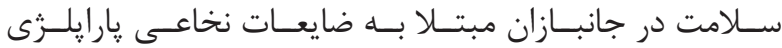

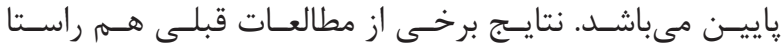

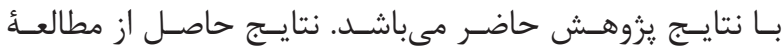

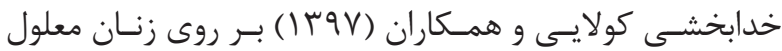

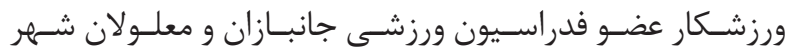

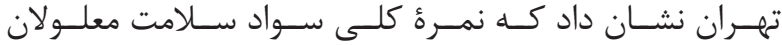

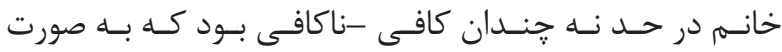

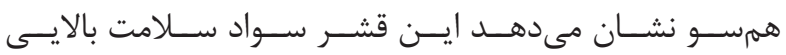

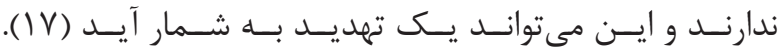

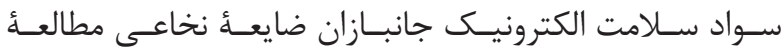

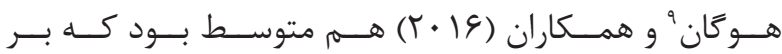

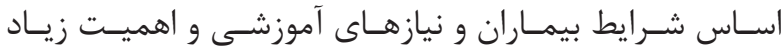

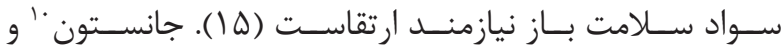

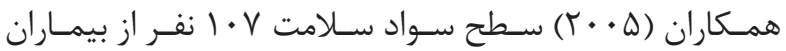

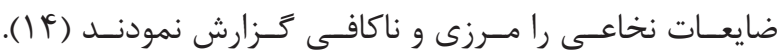

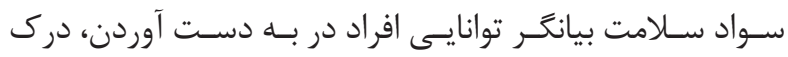

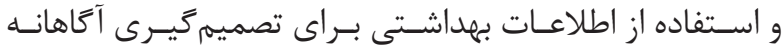

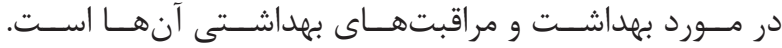

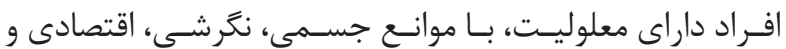

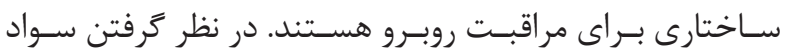

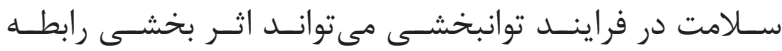

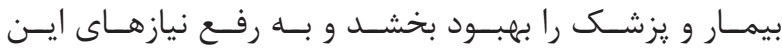

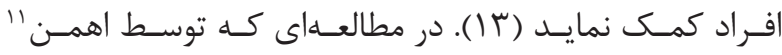

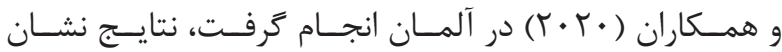

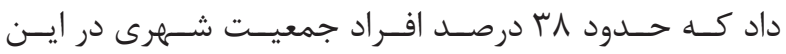

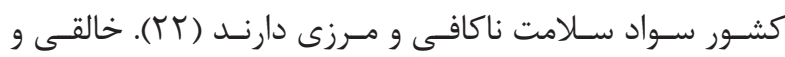

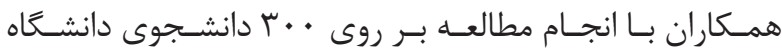

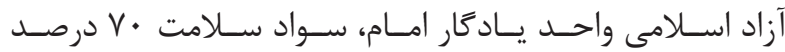

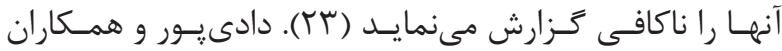

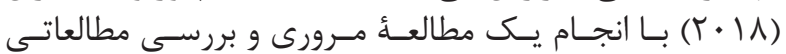

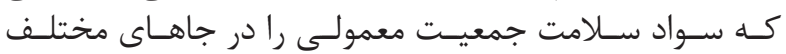

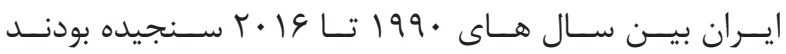

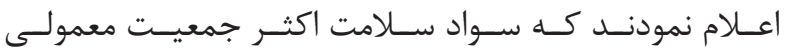

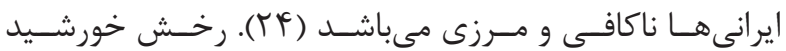

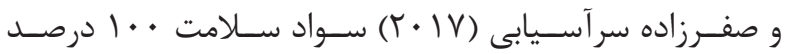

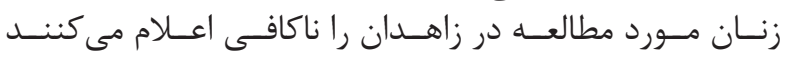

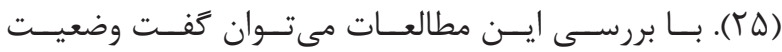

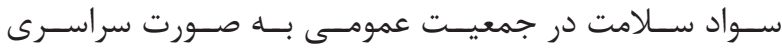

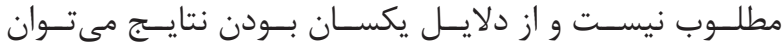

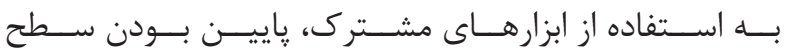

\footnotetext{
${ }^{12}$ Garcia-Codina
}

${ }^{13}$ Protheroe 


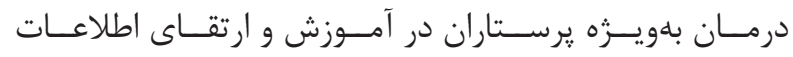

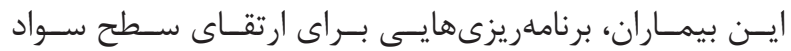

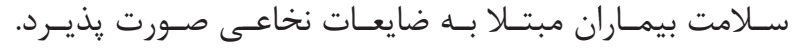
تشكر و قدردانى

ايــن مطالعــه بخشـى از يـــ طـــرح تحققاتـى در دانشـــاه

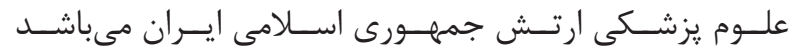

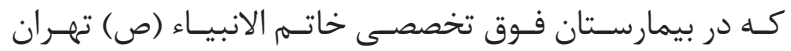

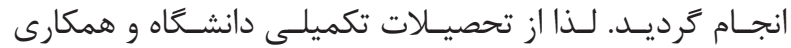

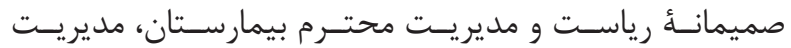

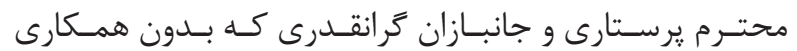

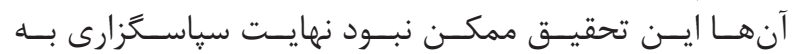

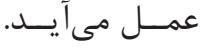

1. Abdullahi M. Evaluation of the use of rehabilitation services in the city of Bam earthquake victims of spinal cord injury. Journal of Qualitative Research in Health sciences. 2011; 11(1,2): 1-6.

2. Kang Y, Ding H, Zhou H, Wei Z, Liu L, Pan D, et al. Epidemiology of worldwide spinal cord injury: a literature review. J Neurorestoratol. 2017; 6: 1-9.

3. Hasanzadehpashing S, Zare H, Alipuor A. The efficacy of stress inculation training (SIT) on resilience, anxiety depression and stress among spinal cord injury (SCI) patients. Journal of Jahrom University of Medical Sciences. 2012; 10(3): 15-25.

4. Economics A. The economic cost of spinal cord injury and traumatic brain injury in Australia. Report by Access Economics for the Victorian Neurotrauma Initiative Canberra: Access Economics. 2009; 31.

5. Seyedoshohadaee M, Barasteh S, Jalalinia F, Eghbali M, Nezami M. The relationship between health literacy and self-care behaviors in patients with type 2 diabetes. Iranian Journal of Nursing Research. 2016;10(4): 43-51.

6. Organization WH. Health literacy: The solid facts. 2013:https://apps.who.int/iris/handle/10665/326432.

7. Naghibi A, Chaleshgar M, Kazemi A, Hosseini M. Evaluation of health literacy level among 18-65 yearold adults in Shahriar, Iran. Journal of health research in community. 2017; 3(2): 17-25.

8. Chen X, Hay JL, Waters EA, Kiviniemi MT, Biddle C, Schofield E, et al. Health literacy and use and trustin health information. Journal of health communication.

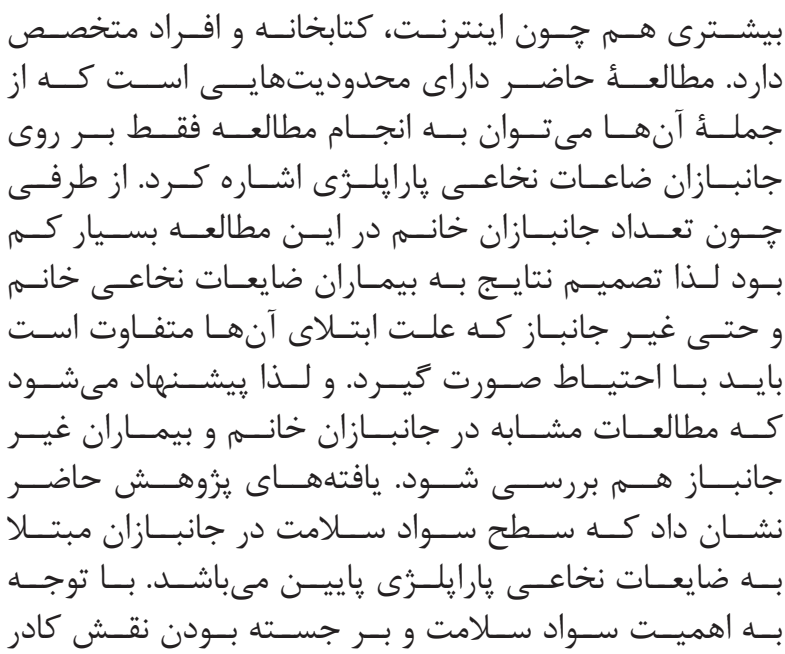

منابع

2018; 23(8): 724-34.

9. Health Literacy [Internet]. 2020.

10. Marzangi A, Gharaaghaji Asl R, Ahangarzadeh Rezaei S. health literacy in heart disease community that covered by health service centers in south of west azarbaijan province. The J Urmia Nurs Midwifery Fac. 2018; 16(1): 45-54.

11. Abdollahi F, Mobadery T, Bajalan Z. Assesse the Health literacy in Multiple Sclerosis patients. Journal of Health Literacy. 2018; 2(4): 266-74.

12. WHO CoSDoH. Closing the gap in a generation: health equity through action on the social determinants of health: final report of the commission on social determinants of health:World Health Organization; 2008.

13. Hahn EA, Magasi SR, Carlozzi NE, Tulsky DS, Wong A, Garcia SF, et al. Health and functional literacy in physical rehabilitation patients. HLRP: Health Literacy Research and Practice. 2017; 1(2): e71-e85.

14. Johnston MV, Diab M, Kim S-S, Kirshblum S. Health literacy, morbidity, and quality of life among individuals with spinal cord injury. The journal of spinal cord medicine. 2005; 28(3): 230-40.

15. Hogan TP, Hill JN, Locatelli SM, Weaver FM, Thomas FP, Nazi KM, et al. Health information seeking and technology use among veterans with spinal cord injuries and disorders. PM\&R. 2016; 8(2): 123-30.

16. Council NR. Review of disability and rehabilitation 


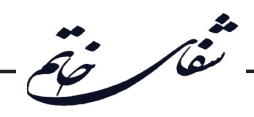

research: NIDRR grantmaking processes and products: National Academies Press; 2012.

17. Khodabakhshi-koolaee A, Flasafinejad MR, Mayeli Khezerloo Z, Mohamadi F. Correlation of Health Literacy with Quality of Life in Athletic Disabled Women. J Educ Community Health. 2018; 5(3): 31-7.

18. Rahmanian M, Ghaffari Targhi M. A survey on health literacy of referred diabetic patients to Yazd diabetes research center. Tolooebehdasht. 2016; 15(3): 176-86.

19. Jalali M. Relationship between health literacy and general health among patients with leukemia before bone marrow transplant. Iranian Journal of Cancer Nursing (ijcn). 2019; 1(1): 1-7.

20. Salehi Z, Nourozitabrizi K, Hosseini M, Akhlaghi M, Rezasoltani P. Health Literacy and its Relationship with Medication Adherence and Quality of Life in Patients with Rheumatoid Arthritis Shariati Hospital Tehran: University of Social Welfare and Rehabilitation Sciences; 2016.

21. Montazeri Ae. Health Literacy for Iranian Adults (HELIA): development and psychometric Properties. Journal of the Iranian Institute for Health Sciences Research. 2014; 13(5): 589-99.

22. Ehmann A, Groene O, Rieger M, Siegel A. The Relationship between Health Literacy, Quality of Life, and Subjective Health: Results of a Cross-Sectional Study in a Rural Region in Germany. International journal of environmental research and public health. 2020; 17(5): 1683.

23. Khaleghi M ASF, Peyman N. The Relationship Between Health Literacy and Health-Related Quality of Life in Students. Iran. J Health Educ Health Promot. 2019; 7(1): 66-73.

24. Dadipoor S, Ramezankhani A, Aghamolaei T, Rakhshani F, Safari-Moradabadi A. Evaluation of health literacy in the Iranian population. Health Scope. 2018; 7(3): e62212.

25. Rakhshkhorshid M, Sarasiyabi A. Investigation of the Health Literacy Level of Women in Reproductive Age as a Public Health Problem in Zahedan. International Journal of Basic Science in Medicine. 2017; 2(2): 101-5.

26. Garcia-Codina $\mathrm{O}$, Juvinyà-Canal $\mathrm{D}$, Amil-Bujan $\mathrm{P}$, Bertran-Noguer C, González-Mestre M, Masachs-Fatjo E, et al. Determinants of health literacy in the general population: Results of the Catalan health survey. BMC Public Health. 2019; 19(1): 1122.

27. Protheroe J, Whittle R, Bartlam B, Estacio E, Clark L, Kurth J. Health literacy, associated lifestyle and demographic factors in adult population of an English city: a cross-sectional survey. Health Expectations. 2017; 20: 112-9.

28. Joveini H, Rohban A, Askarian P, Maheri M, Hashemian M. Health literacy and its associated demographic factors in 18-65-year-old, literate adults in Bardaskan, Iran. Journal of Education and Health Promotion. 2019; 8: 244.

29. Seyedoshohadaee M, Kaghanizade M, Nezami M, Hamedani B, Barasteh S. The relationship between health literacy and general health in patients with type 2 diabetes. Iranian journal of Diabetes and Metabolism. 2016; 15(5): 312-9. 